\title{
Cooperativas de crédito catarinenses: relação entre tamanho e eficiência financeira- operacional
}

\author{
Santa Catarina credit unions: relationship between financial-operational efficiency and size
}

\begin{abstract}
Resumo
O objetivo deste trabalho é mensurar a relação entre tamanho e eficiência financeira-operacional das cooperativas de crédito de Santa Catarina. A literatura apresenta resultados distintos de estudos que buscaram mensurar a influência do tamanho das cooperativas de crédito na sua eficiência. O estudo quantitativo foi estruturado por meio da análise envoltória de dados, da regressão linear simples e da análise de correlação. Os achados da pesquisa identificaram que não há influência do tamanho na eficiência financeira-operacional. Nem mesmo pode-se considerar que exista associação entre a eficiência e o tamanho das cooperativas de crédito e que tais resultados estão em acordo com outros estudos realizados no Brasil. Conclui-se que as práticas de gestão das cooperativas de crédito, por estarem voltadas a atender as necessidades de crédito de seus associados, pode ser um dos fatores que influenciaram os resultados. Outro fator que pode influenciar é a diferença no acesso aos recursos, para operar nas diferentes regiões geográficas.
\end{abstract}

Palavras-chave: Cooperativa de crédito, input, output, análise envoltória de dados, tamanho.

\begin{abstract}
The objective of this paper is to measure the relationship between size and financial-operational efficiency of credit unions in Santa Catarina. The literature presents distinct results from studies that sought to measure the influence of the size of credit unions on their efficiency. The quantitative study was structured by data envelopment analysis, simple linear regression and correlation analysis. The research findings identified that there is no influence of size on financialoperational efficiency. It cannot even be considered that there is an association between the efficiency and size of credit unions and that these results are in agreement with other studies conducted in Brazil. It is concluded that the management practices of credit unions, being focused on meeting the credit needs of their members, maybe one of the factors that influenced the results. Another factor that may influence is the difference in access to resources to operate in different geographic regions.
\end{abstract}

Keywords: Credit Union, input, output, data envelope analysis, size.

Sergio Begnini ${ }^{\mathrm{I}}$, Ieda Margarete Oro ${ }^{\mathrm{II}}$

${ }^{\mathrm{I}}$ Universidade do Oeste de Santa Catarina, sergiobegnini@gmail.com

II Universidade do Oeste de Santa Catarina, iedaoro@unoesc.edu.br 


\section{Introdução}

Atender as demandas apresentadas por seus sócios e atingir a eficiência financeira-operacional, estão entre os objetivos mantidos pelas cooperativas de crédito. Tais objetivos podem estar ligados a busca por manter-se no mercado competitivo. Os resultados financeiros estão ligados às decisões tomadas pelos gestores, considerando variáveis ambientais e organizacionais, e que impactam diretamente na eficiência (CUPERTINO; MARTINEZ; COSTA JR., 2016). As cooperativas, especialmente as mais eficientes, possibilitam a concessão de crédito a praticamente todos seus sócios, promovem a circulação local de recursos, geram sobras que são distribuídas entre os sócios e ainda priorizam o reinvestimento (FERREIRA; GONÇALVES; BRAGA, 2007).

Estudos buscaram entender a relação entre tamanho e eficiência das cooperativas de crédito. Contudo, os resultados mostram contradições, indicando necessidade de maior número de pesquisas para aprofundar o debate e o entendimento. Alguns estudos na Europa (GLASS; MCKILLOP; RASARATNAM, 2010; MARTÍNEZ-CAMPILLO; FERNÂNDEZ-SANTOS, 2017; RAILIENĖ; SINEVIČIENĖ, 2015), na América (WHEELOCK; WILSON, 2013; WIJESIRI; YARON; MEOLI, 2017), (WIJESIRI; YARON; MEOLI, 2017), e da África (WIJESIRI; YARON; MEOLI, 2017), indicaram que grandes cooperativas de crédito apresentam maior eficiência em relação às pequenas. Com resultados distintos, estudos realizados com cooperativas de crédito brasileiras não apresentaram a mesma relação (BRESSAN; LOPES; MENEZES, 2013; GOLLO; SILVA, 2015). Nos casos brasileiros, grandes cooperativas não apresentaram melhor eficiência.

Nota-se então, uma divergência entre os resultados de pesquisas realizadas com cooperativas de crédito brasileiras, em comparação aos resultados com cooperativas estrangeiras, quanto foi mensurada a relação entre tamanho e eficiência. Este estudo busca aprofundar essa discussão, tomando como amostra, cooperativas de crédito do estado de Santa Catarina-Brasil. Embora todos os estudos apresentados neste artigo, tenham analisado questões referentes a eficiência das cooperativas de crédito, nenhum deles utilizou a eficiência financeira-operacional, que é foco da presente pesquisa.

No cenário catarinense, as cooperativas de crédito apresentam relevância, estimulam e contribuem para a diversificação e expansão das atividades nos diversos setores (MATTEI, 2016). Dado o contexto apresentado, surge a pergunta de pesquisa: Qual a relação entre o tamanho e a eficiência financeiraoperacional das cooperativas de crédito em Santa Catarina? Para responder tal questionamento constitui-se como objetivo principal, mensurar a relação entre tamanho e eficiência financeira-operacional das cooperativas de crédito de Santa Catarina.

Para calcular a eficiência financeira-operacional das cooperativas de crédito, foi utilizada a análise envoltória de dados (DEA). Estudos como de Cerqueira, Rezende e Santos (2017), Macedo, Barbosa e Cavalcante (2009) e, Ferreira, Gonçalves e Braga (2007) utilizam e indicam a DEA para realizar o cálculo da eficiência entre várias unidades operacionais. Para calcular o efeito do tamanho na eficiência, foi utilizada regressão linear. Estudos como Glass, Mckiloop e Rasaratnam (2010) e, Railiené e Sinevičienè (2015) utilizam a regressão e atingiram os objetivos propostos. Para o cálculo DEA, foram separadas cinco variáveis, sendo três como inputs e duas como output, com auxílio do Software de Análise Envoltória de Dados (SAED), com modelo Retorno Constante de Escala (CCR) e orientação ao produto.

O artigo contribui com a literatura ao aprofundar o entendimento sobre a relação entre tamanho e eficiência das cooperativas de crédito, abordando a eficiência operacional-financeira, ainda não considerada por outros estudos. Também contribui ao selecionar dados originais e variáveis relevantes para explicar a hipótese da pesquisa, bem como a eficiência operacional-financeira, que não foi utilizada por outros estudos. Ainda contribui com a literatura brasileira, visto que, em comparação com a literatura internacional, no Brasil, carece de estudos sobre a temática de eficiência e tamanho das cooperativas de crédito. Entende-se que, o estudo contribui para que as cooperativas de crédito avaliem as ações na busca por eficiência, desempenho e sustentabilidade no mercado competitivo, considerando o importante papel que possuem junto às pessoas, principalmente aos seus associados. 
O estudo está dividido em cinco seções, além desta introdução. A segunda apresenta o referencial teórico, que aborda questões sobre as cooperativas de crédito e a eficiência dessas em diferentes realidades mundiais. A terceira seção apresenta a metodologia utilizada, e a quarta, os resultados e as discussões. A última parte apresenta as conclusões.

\section{Eficiência em Cooperativas de Crédito}

Dentre as diferentes instituições financeiras existentes, as cooperativas de crédito estão cada vez mais populares e em franco desenvolvimento em vários países, como Estados Unidos, Canadá, Austrália, Japão, França, Alemanha e Itália (MARTÍNEZ-CAMPILLO; FERNÁNDEZ-SANTOS, 2017). Aliado a tais aspectos, surgem pesquisas acadêmicas que buscam entender vários aspectos referentes às cooperativas de crédito. Um desses aspectos é a eficiência, que pode ser estudada a partir de diversas abordagens: financeira; desempenho, produtiva, social, dentre outras. Os estudos relatados na sequência buscaram identificar a existência de relação entre o tamanho da cooperativa de crédito e a sua eficiência.

Na Irlanda, Glass, Mckiloop e Rasaratnam (2010) investigaram fatores que influenciam no desempenho das cooperativas de crédito, utilizando a análise envoltória de dados (DEA) e a regressão, para mensurar. Os autores constaram que um dos elementos chave para garantir a eficiência das cooperativas de crédito, é capacidade de investir em infraestrutura, bem como a regulação e a supervisão, que funcionam de forma adequada, desencadeando menor inadimplência por parte dos usuários (GLASS; MCKILLOP; RASARATNAM, 2010). Os achados da pesquisa indicam que 7\% da amostra atingiram a curva da eficiência, e 93\% opera com algum grau de ineficiência. Aquelas com melhor eficiência possuem baixos níveis de inadimplência, e pagam melhores dividendos aos associados, que as ineficientes. Os dividendos são distribuídos a partir da sobra de excedentes, sendo esperado que tais instituições tenham de atingir, ou aproximar-se ao máximo, da eficiência. Constatou-se ainda que o tamanho das cooperativas influencia diretamente na eficiência financeira-operacional (GLASS; MCKILLOP; RASARATNAM, 2010).

Nos Estados Unidos, Wheelock e Wilson (2013) estudaram as mudanças na eficiência e produtividade das cooperativas de crédito, no período de 1989 a 2006. Destaca-se que nos Estados Unidos, houve substancial aumento no número de associados a partir de 1998, quando as cooperativas passaram a aceitar membros de grupos não relacionados. As cooperativas de crédito, nos Estados Unidos (EUA), detêm $10 \%$ dos depósitos das famílias americanas, e são responsáveis por $9 \%$ dos empréstimos ao consumidor. Entre os resultados, constatou-se que as cooperativas de crédito de grande porte, mantiveram-se eficientes, e as de menor porte apresentaram elevadas quedas na eficiência. Tais quedas estão relacionadas com o aumento no custo de produção (custos operacionais) impactando em declínios nos níveis de produtividade e consequentemente na eficiência produtiva. Os resultados indicam que os avanços tecnológicos e as mudanças advindas das novas regulamentações [que iniciaram na década de 1980], favoreceram as cooperativas de crédito de grande porte, e de certa maneira, contribuíram para a ineficiência das menores (WHEELOCK; WILSON, 2013).

Na Lituânia, Railiené e Sinevičienė (2015) estudaram e discutiram a metodologia de avaliação de desempenho das cooperativas de crédito. Para mensuração utilizaram a análise envoltória de dados e a análise de regressão. As cooperativas de crédito são responsáveis por cerca de $2 \%$ dos ativos financeiros e a participação da população economicamente ativa, nessas empresas, gira em torno de 5,55\%. Embora não seja uma forma expressiva na economia da Lituânia, as cooperativas de crédito apresentam-se como potencial para equilibrar a provisão de serviços financeiros do país (RAILIENĖ; SINEVIČIENĖ, 2015). Constatou-se que as cooperativas de crédito de grande porte são mais eficientes que as pequenas. As principais variáveis influenciadoras foram, maior número de filiais, maior renda líquida, menores taxas de despesas operacionais, e maiores investimentos em marketing. Os autores identificaram que o ambiente de regulação e de avanços tecnológicos, auxilia no desenvolvimento das cooperativas de crédito. A partir dos resultados, em média, as cooperativas podem melhorar a eficiência em $20 \%$ e algumas precisam melhorar algo em torno de $59 \%$ (RAILIENĖ; SINEVIČIENĖ, 2015).

Com base em dados da Ásia, África, América do Sul e Europa, Wijesiri, Yaron e Meoli (2017) investigaram os efeitos da idade e do tamanho na eficiência financeira e social de 420 instituições de micro finanças, dentre estas, 49 cooperativas de crédito. Para mensurar a eficiência utilizaram a análise envoltória de dados. Os resultados do estudo mostraram que, as cooperativas de crédito mais antigas possuem melhor 
desempenho em relação às mais jovens, para alcançar metas financeiras, mas para alcançar os objetivos traçados, possuem maior ineficiência. Constatou-se que as maiores cooperativas tendem a apresentar maior eficiência financeira e social. Tal resultado pode estar relacionado à possibilidade de práticas de economias de escalas, e também, pelo fato de tais cooperativas possuírem maior quantitativo de recursos, pessoal, clientes e crédito. Neste cenário, as cooperativas de crédito parecem estar mais orientadas para o mercado, tanto na captação de clientes (sócios), quanto na prestação de serviços (WIJESIRI; YARON; MEOLI, 2017).

Na Espanha, a partir de dados coletados para o período de 2008-2014, Martínez-Campillo e Fernández-Santos (2017) construíram um índice de eficiência social para as cooperativas de crédito espanholas e buscaram explicações para os resultados, por meio da análise envoltória de dados e da análise de regressão. As cooperativas de crédito espanholas são independentes, sólidas e viáveis com capacidade para cumprir a exigências financeiras e econômicas impostas pela União Europeia, sem a necessidade de recorrer a financiamentos do Estado. As cooperativas, além da eficiência financeira, são caracterizadas por um forte compromisso com a economia e com o desenvolvimento local (MARTÍNEZ-CAMPILLO; FERNÁNDEZ-SANTOS, 2017). A eficiência está atrelada aos pontos de atendimento, revela o estudo. Identificou que, considerando $50 \%$ como um percentual mínimo tolerável, as cooperativas de crédito atingiram 66,4\% de eficiência social. Mesmo no período de crise, as cooperativas de crédito não sofreram tanto quando os bancos tradicionais, em função de não maximizar os lucros, mas satisfazer as necessidades financeiras de seus associados (MARTÍNEZ-CAMPILLO; FERNÁNDEZ-SANTOS, 2017).

No Brasil, a Análise Envoltória de Dados e a técnica econométrica Tobit foi utilizada por Bressan, Lopes e Menezes (2013) para avaliar a performance de cooperativas de crédito filiadas ao sistema de Cooperativas de Crédito do Brasil (Sicoob) no período de 2000 a 2008. Os resultados indicaram que 10\% das cooperativas puderam ser consideradas eficientes. Chegou-se a conclusão que a eficiência técnica, nas cooperativas de crédito filiadas ao Sicoob, não é influenciada pelo tamanho da cooperativa (BRESSAN; LOPES; MENEZES, 2013).

Outro estudo foi realizado por Gollo e Silva (2015) com as 25 maiores cooperativas de crédito brasileiras com dados referentes ao período de 2008 a 2012. A eficiência no desempenho econômicofinanceiro foi medida utilizando a análise multicritério Technique for Orden Perference by Similarity to idela Solution (TOPSIS). Os autores concluíram que o tamanho das cooperativas de crédito não mantém relação com sua eficiência (GOLLO; SILVA, 2015).

\section{Metodologia}

A seleção das cooperativas de crédito ocorreu no site da Organização das Cooperativas do Estado de Santa Catarina (OCESC, 2019). No site, selecionou-se o segmento crédito, e a busca resultou em 57 cooperativas. Neste local foram coletados os dados referentes ao número de funcionários e ao número de associados. Os demais dados foram acessados nos balancetes atinentes a 06/2017, após fazer download dos mesmos, no site do Banco Central do Brasil (BACEN, 2018). Relativo ao nome das cooperativas, em alguns casos, houve divergência entre o nome apresentado no site da OCESC, e no site do BACEN. A solução foi utilizar o CNPJ. Para uma cooperativa não foram encontrados os dados de uma variável, tendo sido excluída. O estudo consistiu de 56 cooperativas de crédito do estado catarinense.

Para o cálculo da eficiência financeira-operacional, utilizou-se a análise envoltória de dados - Data Envelopment Analysis (DEA) - que consiste em uma técnica de programação linear utilizada para avaliar a eficiência técnica relativa de cada Decision Making Units (DMU), dado um conjunto de unidades. Para tanto, são utilizados um ou mais insumos (inputs), e um ou mais produtos (outputs) (CERQUEIRA; DE REZENDE; SANTOS, 2017). Chuang, Chang e Lin (2011) indicam que para medir a eficiência deve-se considerar uma quantia de recursos como entrada e certas quantias de saída, que são os resultados. Uma DMU pode ser uma empresa, um departamento de uma empresa, ou mesmo um sistema produtivo. O cálculo DEA compara uma DMU com toda as outras que participam de determinado estudo, atribuindo um valor entre 0 e 1 para cada uma. As DMUs que obtém valor 1, atingiram a eficiência e quanto mais distante de 1 , menos eficientes são (MACEDO; BARBOSA; CAVALCANTE, 2009).

Dentre os modelos existentes para calcular a eficiência por meio da DEA, os utilizados com maior frequência são o de Retorno Constante de Escala (CRS ou CCR) e o de Retornos Variáveis de Escala (VRS 
ou BCC), sendo que ambos podem ser orientados a insumos ou a produtos (COOPER; SEIFORD; ZHU, 2011). Neste estudo, optou-se pelo modelo CCR para medir a eficiência das cooperativas de crédito, visto que, este modelo, apresenta pontuações com maiores variações, identificando a eficiência global. O método BCC mede a eficiência técnica pura excluindo efeitos de escala (WIJESIRI; YARON; MEOLI, 2017), com orientação ao produto (output) para atingir o máximo de resultados, mantendo constantes as entradas (inputs).

Em face aos resultados indicados pelas pesquisas internacionais, bem como pelas nacionais, nesta pesquisa, optou-se por utilizar variáveis que constituam modelos distintos de avaliação de eficiência, em relação àqueles utilizados nos estudos internacionais e nacionais, a fim de constatar proximidades ou diferenças entre os resultados atingidos. Busca-se assim, aprofundamento na discussão dos resultados, comparando-os com os estudos apresentados na revisão de literatura.

As variáveis no modelo DEA estão identificadas e explicadas no Quadro 1.

Quadro 1 - Variáveis utilizadas no modelo DEA

\begin{tabular}{|c|c|c|c|}
\hline Variável & ID & Significado & Autores \\
\hline $\begin{array}{c}\text { Instalações, } \\
\text { Móveis e } \\
\text { Equipamentos } \\
\text { de Uso (IMEU) }\end{array}$ & Input & $\begin{array}{l}\text { Conjunto de componentes a disposição dos funcionários } \\
\text { para realizar os serviços e o atendimento aos associados. }\end{array}$ & $\begin{array}{l}\text { (CAVA; SALGADO } \\
\text { JUNIOR; BRANCO, } \\
\text { 2016) }\end{array}$ \\
\hline $\begin{array}{l}\text { Ativo Total } \\
\quad(\mathrm{AT})\end{array}$ & Input & São todos os bens e direitos que a cooperativa dispõe. & $\begin{array}{c}\text { (PÉRICO; } \\
\text { REBELATTO; } \\
\text { SANTANA, 2008)* }\end{array}$ \\
\hline $\begin{array}{l}\text { Número de } \\
\text { Funcionários } \\
(\mathrm{NF})\end{array}$ & Input & $\begin{array}{c}\text { Mão de obra que cada cooperativa possui para prestar } \\
\text { serviços e atendimento aos cooperados. }\end{array}$ & $\begin{array}{l}\text { (CAVA; SALGADO } \\
\text { JUNIOR; BRANCO, } \\
\text { 2016) }\end{array}$ \\
\hline $\begin{array}{l}\text { Operações de } \\
\text { Crédito (OC) }\end{array}$ & Output & $\begin{array}{l}\text { Um dos principais objetivos de uma cooperativa de } \\
\text { crédito é realizar empréstimos para minimizar os custos e } \\
\text { maximizar os benefícios ofertados aos cooperados. }\end{array}$ & $\begin{array}{c}\text { (FERREIRA; } \\
\text { GONÇALVES; } \\
\text { BRAGA, 2007; } \\
\text { VILELA; NAGANO; } \\
\text { MERLO, 2007) }\end{array}$ \\
\hline $\begin{array}{l}\text { Resultado } \\
\text { Operacional } \\
(\mathrm{RO})\end{array}$ & Output & $\begin{array}{c}\text { Subtraindo as despesas operacionais, das receitas } \\
\text { operacionais, chega-se ao resultado operacional obtido } \\
\text { pela cooperativa (resultado global líquido). }\end{array}$ & $\begin{array}{l}\text { (FERREIRA; } \\
\text { GONÇALVES; } \\
\text { BRAGA, 2007) }\end{array}$ \\
\hline
\end{tabular}

* Estudo realizado com bancos comerciais. Fonte: Elaboração própria (2019).

Com base na divisão do Instituto Brasileiro de Geografia e Estatísticas (IBGE, 2019), 14 cooperativas de crédito estão presentes na Mesorregião Oeste Catarinense, 11 na Grande Florianópolis, 11 no Vale do Itajaí. Outras 8 situam-se na Mesorregião Serrana, 6 no Sul Catarinense, e 6 no Norte Catarinense. As cooperativas participantes do estudo estão identificadas no Quadro 2.

Quadro 2 - Cooperativas participantes do estudo e respectiva DMU

\begin{tabular}{|l|l|c|l|}
\hline \multicolumn{1}{|c|}{ DMUs } & \multicolumn{1}{|c|}{ Cooperativa } & DMUs & \multicolumn{1}{c|}{ Cooperativa } \\
\hline DMU1 & CREDITRAN & DMU29 & SICOOB SÃO MIGUEL SC \\
\hline DMU2 & SICOOB CENTRAL SC/RS & DMU30 & SICOOB VALE DO VINHO \\
\hline DMU3 & CREDIFIESC & DMU31 & SICOOB CREDIMOC SC \\
\hline DMU4 & COOMARCA & DMU32 & CREDICANOAS \\
\hline DMU5 & CREDELESC & DMU33 & SICOOB CREDICAMPOS SC \\
\hline DMU6 & SICOOB ADVOCACIA & DMU34 & SICOOB/SC - CREDISSERRANA \\
\hline DMU7 & SICOOB CREDISC & DMU35 & CREDICOMIN \\
\hline DMU8 & CREDPOM & DMU36 & SICOOB/SC - CREDIUNIÃO \\
\hline DMU9 & TRANSPOCRED & DMU37 & SICOOB CREDICARU SC \\
\hline DMU10 & UNICRED FLORIANÓPOLIS & DMU38 & SICOOB-CREDIARAUCÁRIA/SC \\
\hline DMU11 & SICOOB TRENTOCREDI SC & DMU39 & SICOOB/SC - CREDISERRA \\
\hline DMU12 & SICOOB - CREDICANOINHAS/SC & DMU40 & SICOOB REDIVALE \\
\hline DMU13 & CREVISC & DMU41 & SICREDI SUL SC \\
\hline DMU14 & SICOOB CEJASCRED & DMU42 & ACENTRA \\
\hline
\end{tabular}




\begin{tabular}{|l|l|l|l|} 
DMU15 & SICREDI NORTE SC & DMU43 & UNICREDI SUL CATARINENSSE \\
\hline DMU16 & SICOOB/SC CREDINORTE & DMU44 & SICOOB/SC CREDIJA \\
\hline DMU17 & SICOOB CREDIPLANALTO SC & DMU45 & SICOOB CREDISULCA SC \\
\hline DMU18 & SICOOB/SC CAÇADOR & DMU46 & CECRED \\
\hline DMU19 & UNICRED OESTE E SERRA & DMU47 & CREDICOR \\
\hline DMU20 & SICOOB MAXICREDITO/SC & DMU48 & VIACREDI \\
\hline DMU21 & SICOOB - TRANSCREDI & DMU49 & UNICRED BLUMENAU \\
\hline DMU22 & SICOOB - CREDIAUC/SC & DMU50 & SICOOB MULTICREDI SC \\
\hline DMU23 & SICOOB CREDIAL/SC & DMU51 & VIACREDI ALTO VALE \\
\hline DMU24 & SICOOB CREDITAPIRANGA SC & DMU52 & UNICRED UNIÃO \\
\hline DMU25 & SICOOB - OESTECREDI/SC & DMU53 & CREDIFOZ \\
\hline DMU26 & SICOOB VALCREDI/SC & DMU54 & SICREDI VALE LITORAL SC \\
\hline DMU27 & SICOOB CREDITAIPU & DMU55 & SICOOB ALTO VALE \\
\hline DMU28 & SICOOB NOROESTE SC & DMU56 & SICOOB/SC CREDIPÉROLA \\
\hline
\end{tabular}

Fonte: Elaboração própria (2019).

Após o cálculo de eficiência das cooperativas, por meio do DEA-SAED, devido à diversidade de resultados apresentados pelas cooperativas consideradas ineficientes, optou-se por criar mais quatro categorias, além da "eficiente", com base na média e no desvio padrão calculado. Para tanto, calculou-se a média e o desvio padrão, sendo respectivamente 0,70282 e 0,19772 . O cálculo e as categorias estão apresentados no Quadro 3.

Quadro 3 - Categorias e os limites dos cálculos para cada categoria

\begin{tabular}{|l|l|l|}
\hline Categorias & limite inferior & limite superior \\
\hline Eficiente & 1,00000 & 1,00000 \\
\hline Ineficiência Baixa & $>\bar{x}_{+1 \square}$ & 0,99999 \\
\hline Ineficiência Média Baixa & $=\bar{x}$ & $=\bar{x}_{+1 \square}$ \\
\hline Ineficiência Média Alta & $=\bar{x}_{-1 \square}$ & $<\bar{x}$ \\
\hline Ineficiência Alta & 0,00000 & $<\bar{x}$ \\
\multicolumn{2}{l}{} \\
\hline
\end{tabular}

Fonte: Elaboração própria (2019).

Após o cálculo da eficiência financeira-operacional, foram realizadas mensurações de efeito, utilizando a regressão linear. Para medir uma possível influência do tamanho da cooperativa em sua eficiência, utilizou-se o escore da eficiência, como variável dependente, e o número de sócios como variável independente. O número de sócios foi utilizado como variável nos estudos de Gorton e Schmid (1999) e Sababu (2015). Posteriormente, para robustez dos resultados, foram realizadas mais seis regressões mantendo o escore da eficiência, como variável dependente e utilizando as seguintes variáveis independentes: instalações, móveis e equipamentos de uso; ativo total; número de funcionários; operações de crédito; resultado operacional; receita operacional. Tais regressões foram necessárias para identificar se havia algum indício de que o tamanho da cooperativa influenciava a eficiência financeira-operacional. Por fim, procedeu-se o cálculo da correlação, por meio do coeficiente de Pearson, para identificar a associação entre as variáveis. Para melhor compreensão da pesquisa, organizou-se a Figura 1. 
Figura 1 - Protocolo da pesquisa

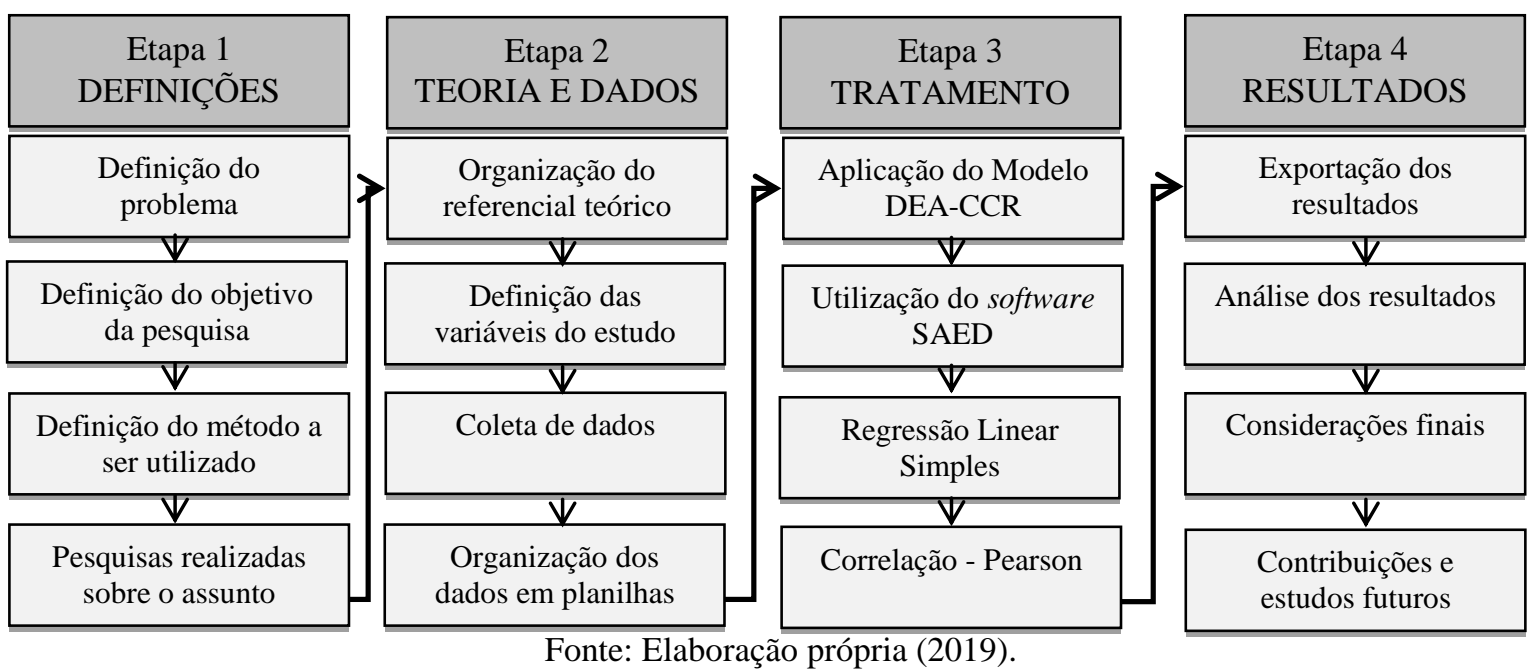

Cada etapa da pesquisa configura-se em um passo importante para sua concretização. Por isso o passo a passo foi delimitado com o cuidado necessário para manter o rigor acadêmico, possibilitando aprofundar o entendimento dos resultados obtidos, por meio de aproximações com os resultados de outros estudos.

\subsection{Hipóteses da pesquisa}

A literatura apresenta diferentes resultados quando pesquisadores buscaram medir a eficiência das cooperativas de crédito, considerando o cenário brasileiro e o cenário internacional. As pesquisas realizadas com cooperativas de crédito da Irlanda, dos Estados Unidos, da Lituânia, da Espanha, da Ásia, África, América do Sul e Europa, apontam que a eficiência dessas cooperativas está relacionada ao tamanho das mesmas. Já os estudos com cooperativas de crédito brasileiras, apontam no sentido contrário, em que a eficiência da cooperativa de crédito não está sendo influenciada por seu tamanho. No caso brasileiro, a eficiência das cooperativas de crédito estaria mais apoiada nas práticas de gestão, do que no seu tamanho. Considerando as duas realidades, e que este estudo abarca cooperativas de crédito brasileiras, formulou-se a seguinte hipótese:

Hipótese central: O tamanho das cooperativas de crédito brasileiras não influencia sua eficiência financeira-operacional.

Hipótese alternativa: O tamanho das cooperativas de crédito brasileiras influencia sua eficiência financeira-operacional.

\section{Resultados e discussões}

Este estudo aborda questões importantes relativas a eficiência de cooperativas de crédito presentes no território do estado de Santa Catarina. Para entender um pouco mais sobre contexto das cooperativas integram a amostra, na sequência, são apresentados alguns dados.

\subsection{Descrição das cooperativas de crédito}

As cooperativas são formadas por um grupo de pessoas que se organizam, a fim de atingir objetivos comuns, em diversos segmentos. Neste estudo, são objetos as cooperativas de crédito do estado de Santa Catarina. Dessas cooperativas, a mais velha é a Sicoob Creditapiranga SC, com 87 anos, e a mais nova é a Viacredi Alto Vale com quase 6 anos. As cooperativas de crédito catarinense são relativamente jovens, possuindo idade média de 24 anos. A Tabela 1 apresenta a estatística descritiva das variáveis utilizadas para o cálculo da eficiência. 
Tabela 1 - Estatística descritiva das variáveis do modelo

\begin{tabular}{lrrrr}
\hline Variáveis & \multicolumn{1}{c}{ Mínimo } & \multicolumn{1}{c}{ Máximo } & \multicolumn{1}{c}{ Média } & Desvio padrão \\
\hline $\begin{array}{l}\text { Input } \\
\text { Instalações, imóveis e }\end{array}$ & 45.181 & 7.367 .860 & 1.693 .532 & 1.888 .847 \\
$\begin{array}{l}\text { equipamentos de uso } \\
\text { Ativo Total }\end{array}$ & 49.494 .337 & 9.942 .905 .290 & 1.338 .783 .491 & 1.952 .688 .584 \\
Número de Funcionários & 10 & 1.274 & 122,50 & 189,75 \\
$\begin{array}{l}\text { Output } \\
\text { Operações de Crédito }\end{array}$ & & & & \\
Resultado Operacional & 7.144 .42 & 1.991 .733 .642 & 170.465 .301 & 285.830 .887 \\
\hline
\end{tabular}

Fonte: Resultados da pesquisa (2019).

Participaram do estudo, cooperativas de crédito de diferentes tamanhos, resultando em maior amplitude dos dados, por exemplo, o ativo total, cuja amplitude foi de R \$ 9.893.410.952, assim como do resultado operacional, que foi de $\mathrm{R} \$ 1$ 1.984.589.216. Outra variável é a quantidade de funcionários, enquanto, uma cooperativa informa possuir 10, outra possui 1.274. A variedade de dados possibilita entender que existem diferenças significativas entre as cooperativas.

\subsection{Eficiência Financeira-Operacional das Cooperativas de Crédito}

As 56 cooperativas de crédito, consideradas neste estudo, foram tratadas por meio do modelo clássico DEA-CCR e o resultado da eficiência financeira-operacional está apresentado na Figura 5. As DMUs foram categorizadas em eficiente, ineficiência baixa, ineficiência média baixa, ineficiência média alta e ineficiência alta. As DMUs que atingiram o resultado 1,00000 são aquelas que chegaram à fronteira da eficiência, portanto, consideradas eficientes. As demais, que não atingiram o mesmo resultado, são consideradas ineficientes.

Conforme Quadro 4, as DMUs 43, 7, 18, 21, 36, 14 e 8 destacam-se como eficientes, representando $12,5 \%$ do total de cooperativas. Na outra extremidade estão as cooperativas $28,3,56,47,55,11$ e 46 , consideradas com alta ineficiência, também representando $12,5 \%$ do total. Outros $41,1 \%$ das cooperativas atingiram ineficiência média alta, 25\% classificaram-se com ineficiência média baixa, e $8,9 \%$ com ineficiência baixa. Os resultados indicando que 07 cooperativas de crédito atingiram a eficiência operacional-financeira, e outras 49 apresentaram algum grau de ineficiência.

As cinco categorias criadas para apresentar os resultados, auxiliam a entender como as cooperativas de crédito, do estado catarinense, estão posicionadas. A distribuição pelas categorias mostra que a maioria delas, precisa dedicar maior atenção para identificar e melhorar aspectos financeiros-operacionais, de modo a atingir melhores resultados. Bressan, Lopes e Menezes (2013) também chegaram a conclusões próximas, indicando a necessidade do desenvolvimento de ações que busquem maximizar os níveis de eficiência das cooperativas de crédito, a partir da maximização dos resultados e da capacidade operacional. 
Quadro 4 - Classificação das DMUs, conforme o resultado de eficiência/ineficiência

\begin{tabular}{|c|c|c|c|c|c|c|c|c|c|c|c|}
\hline DMU & $\begin{array}{c}\text { Resultad } \\
\mathrm{o}\end{array}$ & DMU & $\begin{array}{c}\text { Resultad } \\
\text { o }\end{array}$ & DMU & Resultado & DMU & Resultado & DMU & Resultado & $\mathrm{DMU}$ & $\begin{array}{c}\text { Resultad } \\
\text { o }\end{array}$ \\
\hline \multicolumn{2}{|c|}{ Eficiente } & \multicolumn{2}{|c|}{ Ineficiência Baixa } & \multicolumn{2}{|c|}{$\begin{array}{c}\text { Ineficiência Média } \\
\text { Baixa }\end{array}$} & \multicolumn{2}{|c|}{$\begin{array}{l}\text { Ineficiência Média } \\
\text { Alta }\end{array}$} & \multicolumn{2}{|c|}{$\begin{array}{c}\text { Ineficiência Média } \\
\text { Alta }\end{array}$} & \multicolumn{2}{|c|}{ Ineficiência Alta } \\
\hline $\begin{array}{l}\text { DMU4 } \\
3\end{array}$ & 1,00000 & $\begin{array}{l}\text { DMU3 } \\
7\end{array}$ & 0,97989 & DMU19 & 0,89934 & DMU4 & 0,68479 & DMU20 & 0,58070 & $\begin{array}{l}\text { DMU2 } \\
8\end{array}$ & 0,49786 \\
\hline DMU7 & 1,00000 & $\begin{array}{l}\text { DMU5 } \\
2 \\
\end{array}$ & 0,96841 & DMU6 & 0,89036 & DMU15 & 0,66600 & DMU12 & 0,56814 & DMU3 & 0,47900 \\
\hline $\begin{array}{l}\text { DMU1 } \\
8\end{array}$ & 1,00000 & \begin{tabular}{|l} 
DMU2 \\
7 \\
\end{tabular} & 0,96550 & DMU10 & 0,88009 & DMU16 & 0,66422 & DMU9 & 0,56529 & \begin{tabular}{|l|} 
DMU5 \\
6 \\
\end{tabular} & 0,44700 \\
\hline $\begin{array}{l}\text { DMU2 } \\
1\end{array}$ & 1,00000 & \begin{tabular}{|l} 
DMU4 \\
8 \\
\end{tabular} & 0,96035 & DMU13 & 0,82782 & DMU41 & 0,66065 & DMU22 & 0,55995 & \begin{tabular}{|l} 
DMU4 \\
7
\end{tabular} & 0,43589 \\
\hline $\begin{array}{l}\text { DMU3 } \\
6 \\
\end{array}$ & 1,00000 & $\begin{array}{l}\text { DMU4 } \\
9 \\
\end{array}$ & 0,91802 & DMU39 & 0,80574 & DMU50 & 0,65951 & DMU30 & 0,54242 & $\begin{array}{l}\text { DMU5 } \\
5 \\
\end{array}$ & 0,39634 \\
\hline $\begin{array}{l}\text { DMU1 } \\
4\end{array}$ & 1,00000 & & & DMU51 & 0,79114 & DMU24 & 0,64362 & DMU1 & 0,53702 & \begin{tabular}{|l|} 
DMU1 \\
1
\end{tabular} & 0,39620 \\
\hline \multirow[t]{8}{*}{ DMU8 } & 1,00000 & & & DMU44 & 0,78561 & DMU40 & 0,63984 & DMU45 & 0,53294 & $\begin{array}{l}\text { DMU4 } \\
6 \\
\end{array}$ & 0,11897 \\
\hline & & & & DMU33 & 0,74343 & DMU42 & 0,63878 & DMU35 & 0,52878 & & \\
\hline & & & & DMU17 & 0,73748 & DMU34 & 0,62621 & DMU5 & 0,51998 & & \\
\hline & & & & DMU25 & 0,73555 & DMU31 & 0,62037 & & & & \\
\hline & & & & DMU23 & 0,72639 & DMU54 & 0,60848 & & & & \\
\hline & & & & DMU26 & 0,72046 & DMU38 & 0,60477 & & & & \\
\hline & & & & DMU32 & 0,71914 & DMU53 & 0,59347 & & & & \\
\hline & & & & DMU29 & 0,70382 & DMU2 & 0,58218 & & & & \\
\hline
\end{tabular}

Fonte: Resultados da pesquisa (2019).

Das dez cooperativas com maiores resultados operacionais, nenhuma está entre as sete eficientes ou entre as sete com ineficiência alta. No outro extremo, das dez cooperativas com menores resultados operacionais, duas atingiram a eficiência e duas foram consideradas com ineficiência alta. Tais resultados aproximam-se daqueles encontrados por Gollo e Silva (2015), onde os autores pressupõe que mesmo com as regulações existentes a partir do BACEN, as cooperativas de crédito brasileiras, apresentam desempenhos diferentes entre elas, sendo possível identificar que as estratégias são traçadas pelas próprias cooperativas, a partir do poder de decisão de seus membros.

Verifica-se ainda, que nenhuma das dez cooperativas com maior tempo de atuação, foi considerada eficiente ou com ineficiência alta. Entre as dez com menor tempo de atuação, uma foi considerada eficiente e, uma apresentou ineficiência alta. Tais resultados não convergem para encontrar relação entre idade e eficiência/ineficiência das cooperativas de crédito. Por exemplo, a DMU43 (Cooperativa Unicred Sul Catarinense), considerada a mais eficiente, possui 24,52 anos, sendo que a média de idade das cooperativas é de 24,67 anos. Esses achados diferem daqueles encontrados por Wijesiri, Yaron e Meoli (2017) realizado com "microfinance institutions" onde as empresas com maior tempo de atuação, apresentaram desempenho financeiro superior, em comparação com as de menor tempo de atuação.

Para entender melhor os achados da pesquisa, optou-se por cruzar os resultados da eficiência/ineficiência com os dados das outras variáveis do estudo. Assim, identificou-se que entre as 10 cooperativas com maior Ativo Total (AT), uma ficou classificada com ineficiência alta, 4 com ineficiência média alta, 2 com ineficiência média baixa, 3 com ineficiência baixa, e nenhuma como eficiente. Referente à variável, número de funcionários, entre as dez com maior quantitativo, apresentou-se uma com ineficiência alta e nenhuma como eficiente. Entre as $10 \mathrm{com}$ menor número de funcionários, uma ficou com ineficiência alta e três atingiram a fronteira da eficiência. Referente à variável, operação de crédito, entre as $10 \mathrm{com}$ maiores movimentações, uma ficou classificada com ineficiência alta, cinco com ineficiência média alta, três com ineficiência média baixa, uma com ineficiência baixa e nenhuma como eficiente. Os resultados encontrados, estão de acordo com os apontamentos feitos por Gollo e Silva (2015), de que a eficiência financeira-operacional das cooperativas de crédito, no Brasil, está pouco relacionada com volume de ativos, número de cooperados e receitas, e muito mais com a política de funcionamento e a gestão eficiente dos recursos, efetivada pelos gestores. As análises, a partir dos resultados da DEA, indicaram para a inexistência da relação entre tamanho das cooperativas de crédito em sua eficiência. 
Para mensurar a influência do tamanho na eficiência utilizou-se a regressão linear. A variável independente utilizada foi 'número de sócios' e a variável dependente foi 'escore de eficiência calculado'. A variável independente foi transformada, para atender a necessidade de normalidade dos dados utilizando-se, para tanto, o ln. Os resultados estão indicados na Tabela 2. Os dados atenderam a exigência dos pressupostos de normalidade, homocedasticidade, linearidade, autocorrelação e multicolinearidade.

Tabelas 2 - Estatísticas da regressão

\begin{tabular}{|c|c|c|c|c|c|}
\hline \multicolumn{6}{|c|}{ RESUMO DO MODELO } \\
\hline $\begin{array}{c}\text { Modelo } \\
1\end{array}$ & $\begin{array}{c}\mathbf{R} \\
0,255\end{array}$ & $\begin{array}{c}\text { R quadrado } \\
0,065\end{array}$ & $\begin{array}{c}\mathbf{R} \text { quadrado ajustado } \\
0,048\end{array}$ & \multicolumn{2}{|c|}{$\begin{array}{c}\text { Erro padrão da estimativa } \\
0,19292 \\
\end{array}$} \\
\hline \multicolumn{6}{|c|}{ ANÁLISE DA VARIÂNCIA - ANOVA } \\
\hline Modelo & $\begin{array}{l}\text { Soma dos } \\
\text { quadrados }\end{array}$ & DF & Quadrado médio & $\mathbf{F}$ & Sig \\
\hline Regressão & 0,140 & 1 & 0,140 & 3,768 & 0,057 \\
\hline Residual & 2,010 & 54 & 0,037 & & \\
\hline Total & 2,150 & 55 & & & \\
\hline \multicolumn{6}{|c|}{ COEFICIENTE } \\
\hline \multirow{2}{*}{ Modelo } & \multicolumn{2}{|c|}{ Coeficientes não padronizados } & Coeficiente padronizado & \multirow{2}{*}{$\mathbf{T}$} & \multirow{2}{*}{ Sig } \\
\hline & B & Std error & Beta & & \\
\hline Constant & 0,406 & 0,155 & & 2,623 & 0,011 \\
\hline $\begin{array}{l}\mathrm{N}^{\circ} \text { de } \\
\text { sócios }\end{array}$ & 0,032 & 0,017 & 0,255 & 1,941 & 0,057 \\
\hline
\end{tabular}

Fonte: Resultados da pesquisa (2019).

Nota: variável dependente: Eficiência Operacional-Financeira; variável independente: $\mathrm{N}^{\mathrm{o}}$ de sócios.

A Tabela 2 apresenta os resultados frente à expectativa da não influência do tamanho da cooperativa de crédito na sua eficiência financeira-operacional, conforme Hipótese central. A hipótese foi fundamentada no argumento de que a eficiência encontra maior apoio nas práticas da gestão, que no tamanho da cooperativa. Frente aos resultados, com 95\% de confiança, a hipótese central foi suportada, mostrando que o tamanho da cooperativa de crédito, não influencia sua eficiência financeira-operacional $[\mathrm{F}=3,768]$, p-value $>0,050 ; r^{2}=0,065$.

Todavia por obter maior robustez nos resultados, foram realizadas outras 6 regressões. Para tanto, foi mantida a variável dependente (eficiência financeira-operacional), e alterada a variável independente em cada regressão (instalações, móveis e equipamentos de uso; ativo total; número de funcionários; operações de crédito; resultado operacional; receita operacional). No entanto, nenhuma das regressões mostrou-se significante.

Buscou-se ainda identificar possível existência de associação entre a eficiência calculada e o tamanho das cooperativas. Para tanto foram utilizadas as mesmas variáveis das regressões. Foi calculado o coeficiente de Pearson, cujos resultados estão apresentados na Quadro 5. O valor do coeficiente de correlação pode variar de -1 a +1, sendo que o sinal (+ ou -) indica a direção da relação (HAIR et al., 2009). Quando a correlação entre duas variáveis for perfeitamente positiva o valor será +1 , quando for perfeitamente negativa, o valor será -1, e se o valor for 'zero' não há correlação (FÁVERO et al., 2009). 
Quadro 5 - Correlação entre as variáveis

\begin{tabular}{|c|c|c|c|c|c|c|c|c|}
\hline &  & 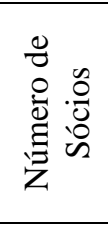 & 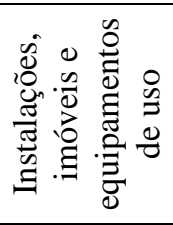 & 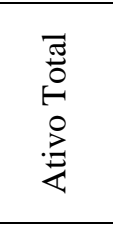 & 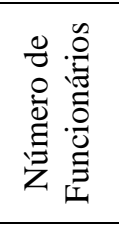 & 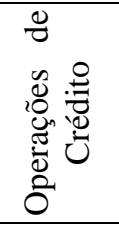 & 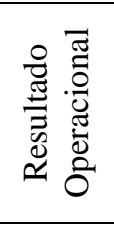 & 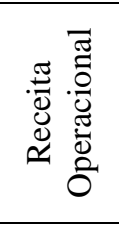 \\
\hline \multirow{3}{*}{$\begin{array}{l}\text { Eficiência } \\
\text { Financeira- } \\
\text { Operacional } \\
\text { (Modelo DEA } \\
\text { CCR output) }\end{array}$} & \multirow[t]{2}{*}{$* 1$} & $* \mathbf{0 , 1 3 2}$ & $* \mathbf{0 , 1 0 0}$ & $* \mathbf{0 , 0 1 7}$ & $* 0,011$ & $* 0,246$ & $* 0,209$ & $* 0-, 016$ \\
\hline & & "0,332 & 0,463 & •0,904 & 0,068 & 0,034 & $\cdot 0,122$ & 0,906 \\
\hline & . 56 & . 56 & . 56 & . 56 & ' 56 & . 56 & $\cdot 56$ & ' 56 \\
\hline Y & & les) & $\overline{\operatorname{tid}}$ & & & & & \\
\hline
\end{tabular}

Fonte: Resultados da pesquisa (2019).

Nota-se que a correlação da variável eficiência financeira-operacional, com as demais, obteve resultados não significativos (maiores que 0,05). A única correlação significativa ocorreu com a variável, operações de crédito. Neste caso, incidiu uma correlação de 0,246 , com $p$-value de 0,034 , ou seja, correlação positiva baixa. Uma possível explicação é o fato de que um dos principais objetivos da cooperativa de crédito é oferecer crédito aos seus associados. Desta forma, as cooperativas podem ofertar mais crédito aos seus associados, na medida em que forem mais eficientes.

Os resultados encontrados neste estudo aproximam-se daqueles identificados por Bressan, Lopes e Menezes (2013) e por Gollo e Silva (2015), de que a eficiência não é influenciada pelo tamanho das cooperativas de crédito brasileiras. Ao mesmo tempo, os resultados são distintos daqueles encontrados por Glass, Mckiloop e Rasaratnam (2010) na Irlanda, por Wheelock e Wilson (2013) nos Estados Unidos, por Railiené e Sinevičienè (2015) na Lituânia, por Wijesiri, Yaron e Meoli (2017) na Ásia, África, América do Sul e Europa, e por Martínez-Campillo e Fernández-Santos (2017) na Espanha, que identificaram o tamanho das cooperativas de crédito, como influenciador da sua eficiência. Os resultados também são, em certa medida, diferentes dos encontrados por Carvalho, Diaz, Bialoskorski Neto e Kalatzis (2015), no Brasil, onde o porte da cooperativa mantém relação com economia de escala, indicando que as maiores possuem mais chances de sobrevivência e de alcançar a eficiência.

Face aos resultados do presente estudo, infere-se que no cenário brasileiro, as cooperativas de crédito apresentam distinção entre os bancos tradicionais, que trabalham na perspectiva de obtenção de lucro sobre as operações (CAVA; SALGADO JUNIOR; BRANCO, 2016), e assim, os maiores atingem maior economia de escala, conseguindo maior eficiência, em comparação aos menores bancos. Já as cooperativas de crédito atuam na perspectiva de atingir quatro propósitos: oferta de empréstimos com menores taxas aos cooperados, oferecer maior possibilidade rentabilidade aos investidores, busca de maximização dos lucros e distribuir igualitariamente entre os associados, os benefícios possíveis (SMITH; CARGILL; MEYER, 1981).

O estudo de Barth (2011) indica que as cooperativas de crédito e os bancos convencionais oferecem os mesmos serviços ao público, estando a diferença principal no fato que, enquanto nos bacos o lucro fica na instituição, nas cooperativas o lucro é dividido entre os sócios. Mesmo assim, os resultados do estudo de Gollo e Silva (2015) indicam que as cooperativas de crédito estão dedicando maiores atenção para atender as necessidades dos associados, enquanto a obtenção de lucros sobre as operações financeiras detém menor atenção. Os resultados do presente estudo também indicam essa mesma realidade, no que tange as cooperativas de crédito catarinenses. Outro fator que pode influenciar os resultados nos estudos é a diferença no acesso aos recursos, para operar nas diferentes regiões geográficas. As cooperativas de crédito ainda podem favorecer o desenvolvimento local, ao ofertar serviços financeiros, com menores taxas e tarifas (JACQUES; GONÇALVES, 2016) em comparação aos bancos tradicionais.

Os resultados também possibilitam identificar as DMUs referências, isto é, o número de vezes que cada DMU eficiente foi considerada modelo para aquelas classificadas como ineficientes. Os gestores de cada DMU ineficiente podem desenvolver planos de melhorias (SILVA; MORETTI; SCHUSTER, 2016) para serem implantados internamente, de modo a atingir a eficiência financeira-operacional. A Figura 2 
mostra as DMUS eficientes e a quantidade de vezes que foram consideradas como benchmark (referência) no modelo.

Figura 2 - Vezes que cada DMU eficiente foi referência para as ineficientes

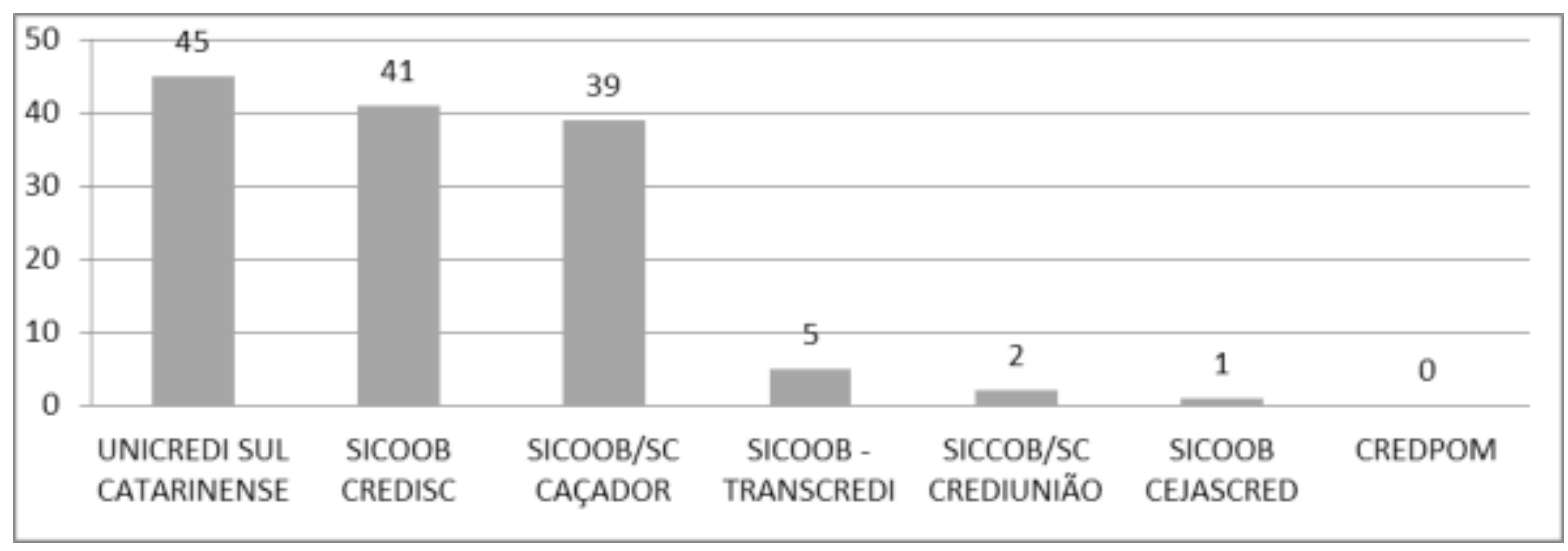

Fonte: Resultados da pesquisa (2019).

As cooperativas Unicredi Sul Catarinense, Sicoob Credisc e Sicoob/SC Caçador, foram benchmark maior quantidade de vezes e maior destaque entre as eficientes. Com base no modelo desta pesquisa, as cooperativas eficientes, especialmente as três que mais vezes foram tidas como referência, demonstram possuir dinamicidade interna, capaz de aproveitar da melhor forma possível, todos os insumos disponíveis, gerando resultados excelentes.

\section{Conclusões}

Em um mercado cada vez mais dinâmico e competitivo, surgem diversas formas de fazer negócio. Uma delas é pela formação de cooperativas, neste estudo, cooperativas de crédito, formadas por pessoas e com interesses comuns. Apresenta-se então um grande, se não o principal, desafio, que é gerir uma empresa, levando em consideração os interesses dos associados, ao mesmo tempo em que, há necessidade de obter sustentabilidade no mercado competitivo de mudanças rápidas.

O propósito deste estudo foi mensurar a influência do tamanho na eficiência financeira-operacional das cooperativas de crédito do estado de Santa Catarina. Para isso utilizou-se a técnica análise envoltória de dados (DEA), para calcular a eficiência e a regressão linear simples para calcular o efeito. O modelo foi elaborado com variáveis que indicam aspectos relacionados ao tamanho das cooperativas de crédito, e que, em conjunto, não haviam sido utilizadas em outros estudos nacionais e internacionais.

Os achados da pesquisa identificaram que não há influência do tamanho na eficiência financeiraoperacional. Nem mesmo pode-se considerar que exista associação entre a eficiência e o tamanho das cooperativas de crédito e que tais resultados estão em acordo com outros estudos realizados no Brasil. Ao mesmo tempo, os resultados se distinguem daqueles de estudos realizados com cooperativas de crédito de outros países, cuja relação é considerada positiva entre o tamanho e a eficiência (GLASS; MCKILOOP; RASARATNAM, 2010; WHEELOCK; WILSON, 2013; RAILIENÉ; SINEVIČIENĖ, 2015; WIJESIRI; YARON; MEOLI, 2017; MARTÍNEZ-CAMPILLO; FERNÁNDEZ-SANTOS, 2017). Com isso, infere-se que as práticas de governança adotadas na gestão das cooperativas de crédito brasileiras, são distintas das praticadas pelas cooperativas de outros países. Outro fator pode ser o ambiente institucional, no qual as cooperativas de crédito brasileiras estão inseridas que, tende a diferenciar-se do ambiente onde as outras cooperativas do mundo estão atuando. Os resultados também levam a compreender que os gestores das cooperativas de crédito brasileiras podem estar mais preocupados com atender as necessidades dos associados, que atingir desempenho econômico-financeiro.

Conclui-se que a maior parte das cooperativas de crédito, neste caso, as cooperativas de crédito catarinenses não atingiram a eficiência e que as demais, estão classificadas em vários graus de ineficiência. Também, que o tamanho das cooperativas de crédito, não influencia a eficiência financeira-operacional. Os 
resultados referem-se a um grupo específico de cooperativas de crédito, e a exclusão de algumas, ou inclusão se outras cooperativas podem resultar em achados distintos.

O estudo apresenta limitações, pela necessidade de escolher algumas das informações (variáveis) existentes, e mesmo pela necessidade de escolher somente um modelo de mensuração. Assim, estudos futuros podem utilizar outras variáveis e/ou outros modelos de análise, como por exemplo, o modelo Tobit, ou a regressão Beta. Destaca-se que poucas pesquisas realizadas no Brasil, buscaram mensurar o tamanho e a eficiência das cooperativas de credito, sendo este um limitador para uma discussão mais ampla. Cabe aos pesquisadores interessados, desenvolver outros trabalhos, em regiões específicas do Brasil, para ampliar e aprofundar as discussões e as conclusões. Sugere-se ainda, desenvolver pesquisas que busquem entender quais ações de gestão distinguem as cooperativas de crédito eficientes, das ineficientes, por meio de entrevistas ou aplicação de questionários com os gestores.

\section{Referências}

BACEN - Banco Central do Brasil. Balancetes. Disponível em: https://www3.bcb.gov.br/informes/relatorios?lingua=pt. Acessado em: 16 out. 2018.

BARTH, M. A cooperativa Prisma sob o enfoque da gestão e das abordagens administrativas: um estudo de caso em uma instituição financeira. Administração Pública e Gestão Social, v.3, n. 3, p. 324-343, 2011.

BRESSAN, V. G. F.; LOPES, A. L. M.; MENEZES, M. R. Análise de eficiência das cooperativas de crédito brasileiras utilizando informações contábeis. 1o Congresso Integrado de Contabilidade, 2013.

CARVALHO, F. L.; DIAZ, M. D. M.; BIALOSKORSKI NETO, S.; KALATZIS, A. E. G. Saída e Insucesso das Cooperativas de Crédito no Brasil: uma análise do risco. Revista Contabilidade \& Finanças, v. 26, n. 67, p. 70-84, 2015.

CAVA, P. B.; SALGADO JUNIOR, A. P.; BRANCO, A. M. D. F. EVALUATION OF BANK EFFICIENCY IN BRAZIL: A DEA APPROACH. RAM. Revista de Administração Mackenzie, v. 17, n. 4, p. 62-84, ago. 2016.

CERQUEIRA, J. D. S.; DE REZENDE, A. A.; SANTOS, C. E. R. Os efeitos dos royalties da mineração sobre a promoção do desenvolvimento econômico dos municípios baianos: uma análise para o período entre 2009 e 2011 por meio da abordagem DEA. RACE - Revista de Administração, Contabilidade e Economia, v. 16, n. 2, p. 603, 30 ago. 2017.

CHUANG, C. L.; LIN, R. H. An efficiency data envelopment analysis model reinforced by classification and regression tree for hospital performance evaluation. Journal of Medical Systems, v.35, n. 5, p. 1075-1083, 2011.

COOPER, W. W.; SEIFORD, L. M.; ZHU, J. Handbook on Data Envelopment Analysis. Boston: Springer US, 2011.

CUPERTINO, C. M;, MARTINEZ, A. L.; COSTA JR., N. C. A. Consequências para rentabilidade futura com o gerenciamento de resultados por meio de atividades operacionais reais. Revista de Contabilidade e Finanças, v.27, n.71, p. 232-242, 2016.

FÁVERO, L. P. et al. Análise de dados: modelagem multivariada para tomada de decisões. Rio de Janeiro: Elsevier, 2009. 
FERREIRA, M. A. M.; GONÇALVES, R. M. L.; BRAGA, M. J. Investigação do desempenho das cooperativas de crédito de Minas Gerais por meio da Análise Envoltória de Dados (DEA). Economia Aplicada, v. 11, n. 3, p. 425-445, set. 2007.

GLASS, J. C.; MCKILLOP, D. G.; RASARATNAM, S. Irish credit unions: Investigating performance determinants and the opportunity cost of regulatory compliance. Journal of Banking \& Finance, v. 34, n. 1, p. 67-76, jan. 2010.

GOLLO, V.; SILVA, T. P. DA. Eficiência global no desempenho econômico-financeiro de cooperativas de crédito brasileiras. Revista de Contabilidade e Organizações, v. 25, p. 43-55, 2015.

GORTON, G.; SCHMID, F. Corporate governance, ownership dispersion and efficiency: Empirical evidence from Austrian cooperative banking. Journal of Corporate Finance, v. 5, p. 119-140, 1999. HAIR, J. F. et al. Análise multivariada de dados. 6. ed. Porto Alegre: Bookman, 2009.

IBGE - Instituto Brasileiro de Geografia e Estatística. Divisão regional do Brasil em mesorregiões e microrregiões geográficas. Disponível em: https://biblioteca.ibge.gov.br/visualizacao/monografias/GEBIS\%20\%20RJ/DRB/Divisao\%20region al_v01.pdf. Acessado em 2 fev. 2019.

JACQUES, E. R.; GONÇALVES, F. O. Cooperativas de crédito no Brasil: evolução e impacto sobre a renda dos municípios brasileiros. Economia e Sociedade, v. 25, n. 2, p. 486-509, 2016.

MACEDO, M. A. S.; BARBOSA, A. C. T. A. M.; CAVAlCANTE, G. T. Desempenho de Agências Bancárias no Brasil: aplicando Análise Envoltória de Dados (DEA) a indicadores relacionados às perspectivas do BSC. Revista Economia \& Gestão, v. 9, n. 19, p.65-84, 2009.

Mattei, L. A importância do sistema familiar de produção no estado de Santa Catarina. Revista Necat, v. 5, n. 9, 2016.

MARTÍNEZ-CAMPILLO, A.; FERNÁNDEZ-SANTOS, Y. What About the Social Efficiency in Credit Cooperatives? Evidence from Spain (2008-2014). Social Indicators Research, v. 131, n. 2, p. 607-629, 20 mar. 2017.

OCESC - Organização das Cooperativas do Estado de Santa Catarina. Busca por cooperativa. 2019. Disponível em: http://www.ocesc.org.br/cooperativas_busca. Acessado em: 05 jun. 2019.

PÉRICO, A. E.; REBELATTO, D. A. DO N.; SANTANA, N. B. Eficiência bancária: os maiores bancos são os mais eficientes? Uma análise por envoltória de dados. Gestão \& Produção, v. 15, n. 2, p. 421-431, ago. 2008.

RAILIENĖ, G.; SINEVIČIENĖ, L. Performance Valuation of Credit Unions Having Social and Self-sustaining Aim. Procedia - Social and Behavioral Sciences, v. 213, p. 423-429, dez. 2015.

Sababu, B. M. The impact of demographic and socioeconomic factors on cooperatives performance. European Journal of Business Management, v. 2, n. 6, p. 49-56, 2015.

SILVA, M. Z. DA; MORETTI, B. R.; SCHUSTER, H. A. Avaliação da Eficiência Hospitalar por Meio da Análise Envoltória de Dados. Revista de Gestão em Sistemas de Saúde, v. 5, n. 2, p. 100114, 1 dez. 2016.

SMITH, D. J.; CARGILL, T. F.; MEYER, R. A. CREDIT UNIONS: An Economic Theory of a Credit Union. The Journal of Finance, v. 36, n. 2, p. 519-528, maio 1981.

VILELA, D. L.; NAGANO, M. S.; MERLO, E. M. Aplicação da análise envoltória de dados em cooperativas de crédito rural. Revista de Administração Contemporânea, v. 11, n. spe2, p. 99-120, 2007. 
WHEELOCK, D. C.; WILSON, P. W. The evolution of cost-productivity and efficiency among US credit unions. Journal of Banking \& Finance, v. 37, n. 1, p. 75-88, jan. 2013.

WIJESIRI, M.; YARON, J.; MEOLI, M. Assessing the financial and outreach efficiency of microfinance institutions: Do age and size matter? Journal of Multinational Financial Management, v. 40, p. 63-76, jun. 2017. 